\title{
EQUIVARIANT FIXED POINT INDEX AND FIXED POINT TRANSFER IN NONZERO DIMENSIONS
}

\author{
CARLOS PRIETO AND HANNO ULRICH
}

\begin{abstract}
Dold's fixed point index and fixed point transfer are generalized for certain coincidence situations, namely maps which change the "equivariant dimension." Those invariants change the dimension correspondingly. A formula for the index of a situation over a space with trivial group action is exhibited. For the transfer, a generalization of Dold's Lefschetz-Hopf trace formula is proved.
\end{abstract}

\section{INTRODUCTION}

0.0. For fixed point situations

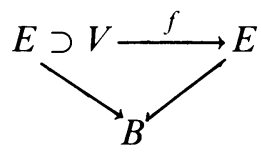

where $E \rightarrow B$ is an $\operatorname{ENR}_{B}, V$ is open and $f$ is compactly fixed, i.e. Fix $(f)=$ $\{v \in V \mid f(v)=v\} \rightarrow B$ is proper, Dold defined in $\left[\mathrm{Do}_{2}\right]$ a fixed point index $I(f)$ which lives in the zeroth group $h^{0}(B)$ for a generalized cohomology theory $h$. In $\left[\mathrm{Do}_{3}\right]$ he defined a fixed point transfer $\check{\tau}_{f}: \check{h}^{*}(\operatorname{Fix}(f)) \rightarrow h^{*}(B)$, which is related to the index by the formula $\check{\tau}_{f}(1)=I(f), 1 \in \check{h}^{0}(\operatorname{Fix}(f))$, where $\check{h}$ means "čechification" of $h$. In this paper we consider more general situations, namely if $G$ is a compact Lie group we take

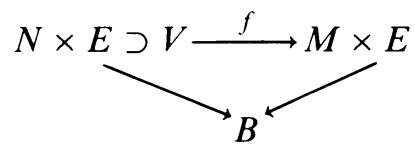

where $E \rightarrow B$ is a $G$ - $\mathrm{ENR}_{B}, M, N$ are real $G$-modules, $V$ is open and $G$ invariant and $f$ is equivariant and compactly fixed, i.e. $\operatorname{Fix}(f)=\{(y, e) \in$ $V \mid f(y, e)=(0, e)\} \rightarrow B$ is proper.

In $\S 1$ we construct a transfer $\tau_{f}^{X}: h_{G}^{*}(X) \rightarrow h^{*+\rho}(B)$ for $h_{G}^{*}$ any $R O(G)$ graded (multiplicative) cohomology theory and $\rho=[M]-[N] \in R O(G)$, where

Received by the editors October 10, 1989.

1980 Mathematics Subject Classification (1985 Revision). Primary 54H25, 55M02, 55M25; Secondary $19 \mathrm{~L} 47,55 \mathrm{~N} 15,55 \mathrm{~N} 91,55 \mathrm{P} 42,55 \mathrm{P} 91,55 \mathrm{Q} 91$.

Key words and phrases. Degree, fixed point index, transfer, equivariant fixed point theory, $R O(G)$-graded equivariant cohomology theory, Lefschetz-Hopf formula. 
$X$ is any ( $G$-invariant) neighborhood of $\operatorname{Fix}(f)$. If as Dold does, we define $\check{h}_{G}^{*}(\operatorname{Fix}(f))=\lim _{X} h_{G}^{*}(X)$ we have also $\check{\tau}_{f}: \check{h}_{G}^{*}(\operatorname{Fix}(f)) \rightarrow h_{G}^{*+\rho}(B)$.

In $\S 2$ we study the index $I(f) \in h_{G}^{\rho}(B)$, which relates to the transfer through the formula $I(f)=\check{\tau}_{f}(1)=\tau_{f}^{X}(1)$ for $1 \in h_{G}^{0}(X)$, and measures the existence of fixed points, namely of elements of $\operatorname{Fix}(f)$. From the properties of the transfer, we see that the index has the expected properties. There we exhibit a formula which in the case of a trivial $G$-action on $B$ describes $I(f)$ in terms of the indices of the restrictions of $f$ to the (finitely many) parts of $V$ of only one orbit type.

In $\S 3$ we prove a Lefschetz-Hopf theorem which generalizes the trace formula of $\left[\mathrm{Do}_{3}\right]$. There the transfer for certain fixed point situations evaluated in suitable elements is computed as a trace of an adequate endomorphism, which changes dimensions. To that end we resume all theoretical background which Dold already formulated in $\left[\mathrm{Do}_{3}, 6\right]$. In fact, after stating the theorem conveniently, most of Dold's proof goes through in this case. As a matter of fact, a special case of this proof can be found in the second edition of Dold's book [Do $\mathrm{Do}_{1}$.

To finish the paper we state in a very succinct form the relationship of these ideas to Becker-Gottlieb's duality-transfer [BG]. They generalize work therein made by Waner [Wa].

Literature on $R O(G)$-graded equivariant cohomology would be [Ko, LMM, LMS or $\mathrm{Pr}_{2}$ ], for instance.

As a last comment I would like to mention that Ize, Massabò and Vignoli have developed in [IMV], a degree for equivariant maps, which, in their finite dimensional case, can easily be obtained from our index, thus, inheriting also the corresponding properties from those of the index. They succeed in computing it in certain cases, when the group acting is the circle $\mathbf{S}^{1}$.

\section{THE EQUIVARIANT TRANSFER}

In this section we shall give the definition and properties of the transfer.

1.0. Let $G$ be a compact Lie group and $B$ a metric space with a continuous action of $G$-a metric $G$-space, for short-(alternatively, we may assume as in [Ul] that $B$ instead of metric is paracompact, and its topology is compactly generated).

1.1. Definition. Let $E$ be a $G$-space and $p: E \rightarrow B$ a continuous $G$-map. $p: E \rightarrow B$ is called a G-euclidean neighborhood retract over $B$, a $G-E^{-} R_{B}$, if there exist a $G$-module $L$, i.e. a finite dimensional real representation of $G$, a $G$-invariant open set $U \subset L \times B$ and $G$-maps $i: E \rightarrow U, r: U \rightarrow E$ over $B$, such that $\operatorname{proj}_{B} \circ i=p, p \circ r=\operatorname{proj}_{B}$ and $r \circ i=\mathrm{id}_{E}$. (For general properties of $G-\mathrm{ENR}_{B} \mathrm{~s}$ see [Ul].) 
We shall be interested in the following coincidence situations:

$$
\begin{array}{ccc}
N \times E \supset V & \stackrel{f}{\rightarrow} & M \times E \\
p \circ \operatorname{proj}_{E} \searrow & & \swarrow p \circ \operatorname{proj}_{E}
\end{array}
$$

where $p: E \rightarrow B$ is a $G$ - $\mathrm{ENR}_{B}, M$ and $N$ are $G$-modules, $V$ is an invariant open subset of $N \times E$ and $f$ is compactly fixed, i.e. if we consider the set (of coincidences) $\operatorname{Fix}(f)=\{(y, e) \in V \mid f(y, e)=(0, e)\}$, then the restriction map $\operatorname{Fix}(f) \rightarrow B$ is proper, that is, it is closed and its fibers are compact. Let $h_{G}^{*}$ be an $R O(G)$-graded $G$-equivariant cohomology theory; e.g. $K_{G}=$ equivariant $K$-theory, $G$-FIX ${ }^{*}=$ equivariant stable cohomotopy $\left[\mathrm{Pr}_{2}\right], H_{G}^{*}=$ ordinary $R O(G)$-graded cohomology [LMM], etc. In order to define the transfer, we shall first consider a special case, namely, we assume $E=L \times B$.

Since $F=\operatorname{Fix}(f) \subset V \subset N \times L \times B$ is proper, then it has a tubular neighborhood (see $\left[\mathrm{Pr}_{3}\right]$ ), namely there exists a $G$-invariant function

$$
\rho: B \rightarrow \mathbf{R}^{+}=(0,+\infty)
$$

such that $F \subset \mathbf{B}_{\rho}=\{(y, b) \in N \times L \times B \mid\|y\|<\rho(b)\}$, where we may assume without loss of generality that $N \times L$ is provided with an invariant metric.

Let, moreover, $X$ be any $G$-invariant open neighborhood of $F=\operatorname{Fix}(f)$ in $V$ and consider the following sequence of $G$-maps (of pairs):

$$
\begin{aligned}
& (M \times L, M \times L-0) \times X \\
& (X, X-F) \quad \stackrel{(i-f, \text { id })}{\longrightarrow}(M \times E, M \times E-0) \times{ }_{B} X \\
& \downarrow \text { (1) } \\
& \left(N \times E, N \times E-\mathbf{B}_{\rho}\right) \rightarrow(N \times E, N \times E-F) \\
& \downarrow \text { (2) } \\
& (N \times E, N \times E-0) \\
& (N \times L, N \times L-0) \times B
\end{aligned}
$$

After applying $h_{G}^{*}$, the vertical arrows (1) and (2) become isomorphisms, since (1) is an excision and (2) a homotopy equivalence (on the second space). Thus we obtain the following homomorphism:

$$
h_{G}^{*}((M \times L, M \times L-0) \times X) \rightarrow h_{G}^{*}((N \times L, N \times L-0) \times B) .
$$

Since $h_{G}^{*}((M \times L, M \times L-0) \times X) \cong h_{G}^{*-([M]+[L])}(X)$, and correspondingly, $h_{G}^{*}((N \times L, N \times L-0) \times B) \cong h_{G}^{*-([N]+[L])}(B)$ (see e.g. $\left.\left[\operatorname{Pr}_{2}\right]\right), 1.4$ determines (after reindexing) homomorphisms

$$
\tau_{f}^{X}: h_{G}^{*}(X) \rightarrow h_{G}^{*+[M]-[N]}(B)
$$

called transfers of $f$. In particular, we have in one extreme the transfer

$$
\tau_{f}: h_{G}^{*}(E) \cong h_{G}^{*}(N \times E) \rightarrow h_{G}^{*+[M]-[N]}(B)
$$


obtained by composing (any) $\tau_{f}^{X}$ with the restriction $h_{G}^{*}(E) \cong h_{G}^{*}(N \times E) \rightarrow$ $h_{G}^{*}(X)$, as well as, after passing to the limit with respect to $X\left(\mathrm{cf} .\left[\mathrm{Do}_{3}\right]\right)$ the minimal transfer

$$
\check{\tau}_{f}: \check{h}_{G}^{*}(\operatorname{Fix}(f)) \rightarrow h_{G}^{*+[M]-[N]}(B)
$$

through which all other transfers factor, that is, $\tau_{f}^{X}=\check{\tau}_{f}$ ores, where res: $h_{G}^{*}(X)$ $\rightarrow \check{h}_{G}^{*}(\operatorname{Fix}(f))$ is the map into the limit. This shows in particular that

$$
\operatorname{Fix}(f)=\phi \Rightarrow \tau_{f}^{X}=0 \quad \text { for every } X \subset N \times E .
$$

(This follows also directly from (1.3).)

There is also a relative transfer for any invariant $A \subset B$, such that $\operatorname{Fix}(f)$ does not meet any of the fibers over $A$, i.e. $\left(N \times E_{A}\right) \cap \operatorname{Fix}(f)=\phi$ if $E_{A}=$ $p^{-1}(A) \subset E$. It is

$$
\tau_{f}^{X}: h_{G}^{*}(X) \rightarrow h_{G}^{*+[M]-[N]}(B, A)
$$

for every $X$ and (1.5) factors through it

The transfer has the following properties:

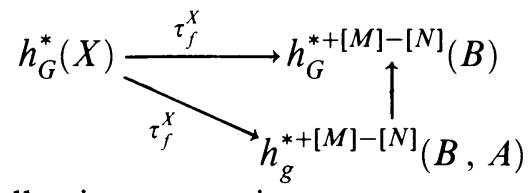

1.10. Units. If $s: B \rightarrow E$ is a section of $p$ and $k: E \rightarrow M \times E$ is defined by $k(e)=(0, s p(e))$, then $\tau_{k}=s^{*}: h_{G}^{*}(s B) \cong h_{G}^{*}(B)$ if $M=0$ and $\tau_{k}=0$ if $M \neq 0$.

1.11. Pullbacks. Let $B^{\prime}$ be metric and $\beta: B^{\prime} \rightarrow B$ be a continuous G-map. If $f$ is compactly fixed over $B$, then its pullback over $\beta, f^{\prime}: V^{\prime} \rightarrow M \times E^{\prime}$, has a transfer $\tau_{f^{\prime}}$, which makes the diagram

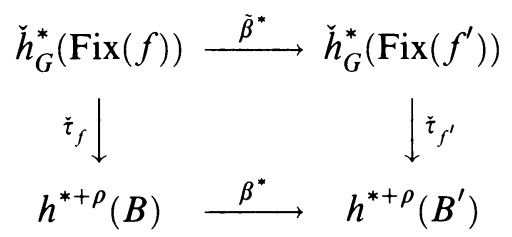

commutative, where $\tilde{\beta}: \operatorname{Fix}\left(f^{\prime}\right) \rightarrow \operatorname{Fix}(f)$ is induced by $\beta$ and $\rho=[M]-[N] \in$ $R O(G)$.

As a special case of 1.11 , we obtain

1.13. Homotopy. If $g: W \rightarrow N \times E, W \subset M \times E$, is compactly fixed over $B \times I$, where $E \rightarrow B \times I$ is a $G-\mathrm{ENR}_{B \times I}$ and if $g_{t}: W_{t} \rightarrow N \times E_{t}$ is the part of $g$ over $B \times\{t\} \approx B$, then the following diagram commutes:

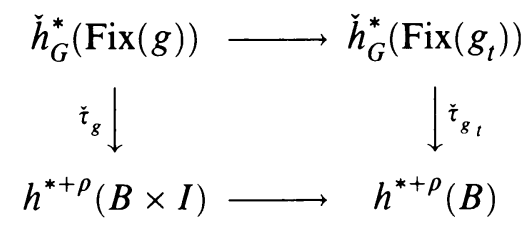


where $\rho=[M]-[N] \in R O(G)$ and the isomorphism at the bottom is induced by the obvious homotopy equivalence between $B$ and $B \times I$.

1.14. Stability. If $P$ is a G-module and $e: P \rightarrow P$ is a constant map (e.g. $e=0)$, then $e \times f: P \times V \rightarrow P \times M \times E$ is compactly fixed and, after identifying $\operatorname{Fix}(e \times f)=\{0\} \times \operatorname{Fix}(f)$ with $\operatorname{Fix}(f)$, we have $\tau_{e \times f}=\tau_{f}: h_{G}^{*}(\operatorname{Fix}(f)) \rightarrow$ $h_{G}^{*+\rho}(B)$.

There is also a commutativity property for the transfer, which allows us to pass to general $G$ - $\mathrm{ENR}_{B} \mathrm{~s}$. It is as follows:

1.15. Commutativity. Let $E=L \times B \rightarrow B$ and $E^{\prime}=L^{\prime} \times B \rightarrow B$ and let $U \subset N \times E$, and $U^{\prime} \subset E^{\prime}$ be open and invariant. If $f: U \rightarrow M \times E^{\prime}$ and $g: U^{\prime} \rightarrow E$ are G-maps over $B$ such that

$$
N \times E \supset f^{-1}\left(M \times U^{\prime}\right) \stackrel{\left(1_{M} \times g\right) f}{\longrightarrow} M \times E
$$

is compactly fixed, then also

$$
N \times E^{\prime} \supset\left(1_{N} \times g\right)^{-1}(U) \stackrel{f\left(1_{N} \times g\right)}{\longrightarrow} M \times E^{\prime}
$$

is compactly fixed, and if $\rho=[M]-[N] \in R O(G)$, the transfer

$$
\check{\tau}_{(1 \times g) f}: \check{h}_{G}^{*}(\operatorname{Fix}((1 \times g) f)) \rightarrow h_{G}^{*+\rho}(B)
$$

coincides with the composition

$$
\check{h}_{G}^{*}(\operatorname{Fix}((1 \times g) f)) \cong \check{h}_{G}^{*}(\operatorname{Fix}(f(1 \times g))) \stackrel{\check{t}_{f(1 \times g)}}{\longrightarrow} h_{G}^{*+\rho}(B),
$$

where the isomorphism is given by a homeomorphism.

Proof. There is a homeomorphism

$$
N \times E \supset \operatorname{Fix}((1 \times g) f) \approx \operatorname{Fix}(f(1 \times g)) \subset N \times E^{\prime}
$$

given by $(y, e) \mapsto\left(y, f_{2}(y, e)\right)$ if $f(y, e)=\left(f_{1}(y, e), f_{2}(y, e)\right) \in M \times E^{\prime}$. Its inverse is given by $\left(y, e^{\prime}\right) \rightarrow\left(y, g\left(e^{\prime}\right)\right)$, as one may easily check.

Let

$$
N \times E \times_{B} E^{\prime} \supset U \times_{B} U^{\prime} \stackrel{\vartheta}{\rightarrow} M \times E \times_{B} E^{\prime}
$$

be given by $\gamma\left(u, u^{\prime}\right)=\left(f_{1}(u), g\left(u^{\prime}\right), f_{2}(u)\right)$, where $f(u)=\left(f_{1}(u), f_{2}(u)\right) \in$ $M \times E^{\prime}$. We use the homotopy invariance to show that $\check{\tau}_{\gamma}=\check{\tau}_{(1 \times g) f}$ and that $\check{\tau}_{\gamma}=\check{\tau}_{f(1 \times g)}$.

We use first the deformation

$$
\gamma_{t}\left(u, u^{\prime}\right)=\left(f_{1}(u), \operatorname{tg} f_{2}(u)+(1-t) g\left(u^{\prime}\right), f_{2}(u)\right) .
$$

A fixed point $\left(u, u^{\prime}\right)$ of $\gamma_{t}$ satisfies $\gamma_{t}\left(u, u^{\prime}\right)=\left(0, u_{2}, u^{\prime}\right)\left(u=\left(u_{1}, u_{2}\right) \in\right.$ $N \times E)$, i.e. $f_{1}(u)=0, u^{\prime}=f_{2}(u)$ and $u_{2}=\operatorname{tg} f_{2}(u)+(1-t) g\left(u^{\prime}\right)$, hence $u_{2}=g\left(u^{\prime}\right)=g f_{2}(u)$. Therefore

$$
\operatorname{Fix}\left(\gamma_{t}\right)=\left\{\left(u, u^{\prime}\right) \mid u \in \operatorname{Fix}((1 \times g) f), u^{\prime}=f_{2}(u)\right\} \approx \operatorname{Fix}((1 \times g) f),
$$


thus it lies properly over $B$. Consequently $\check{\tau}_{\gamma}=\check{\tau}_{\gamma_{0}}=\check{\tau}_{\gamma_{1}}$. But

$$
\gamma_{1}\left(u, u^{\prime}\right)=\left(f_{1}(u), g f_{2}(u), f_{2}(u)\right)=\left((1 \times g) f(u), f_{2}(u)\right),
$$

thus $\gamma_{1}$ is the restriction of

$$
\begin{gathered}
N \times E \times{ }_{B} E^{\prime} \supset U \times{ }_{B} E^{\prime} \stackrel{\delta}{\rightarrow} N \times E \times{ }_{B} E^{\prime}, \\
\delta\left(u, e^{\prime}\right)=\left(\left(1_{M} \times g\right) f(u), f_{2}(u)\right) .
\end{gathered}
$$

But $\delta$ may be deformed by

$$
\delta_{t}\left(u, u^{\prime}\right)=\left(\left(1_{M} \times g\right) f(u),(1-t) f_{2}(u)\right) .
$$

A fixed point of $\delta_{t}$ satisfies $f_{1}(u)=0, g f_{2}(u)=u,(1-t) f_{2}(u)=u^{\prime}$, so that $\bigcup_{t} \operatorname{Fix}\left(\delta_{t}\right)$ is the image of

$$
\begin{gathered}
\operatorname{Fix}((1 \times g) f) \times[0,1] \rightarrow U \times{ }_{B} E^{\prime}, \\
(u, t) \mapsto\left(u,(1-t) f_{2}(u)\right)
\end{gathered}
$$

whence it lies properly over $B$. Hence $\check{\tau}_{\left(1_{M} \times g\right) f}=\check{\tau}_{\delta_{0}}=\check{\tau}_{\delta_{1}}=\check{\tau}_{\gamma}$.

By the symmetry of $\gamma$, we also have $\check{\tau}_{\gamma}=\check{\tau}_{f\left(1_{N} \times g\right)}$.

We are now in position to state the

1.16. Proposition and Definition. If $p: E \rightarrow B$ is any $G-\mathrm{ENR}_{B}, M$ and $N$ are $G$-modules and $V \subset N \times E$ is open and invariant, then every continuous map $f: V \rightarrow M \times E$ admits a decomposition

$$
f: V \stackrel{1_{N} \times \alpha}{\longrightarrow} U \stackrel{\beta}{\longrightarrow} M \times E
$$

over $B$, where $U$ is open in $N \times L \times B$ for some $G$-module $L$. If $f$ is compactly fixed, then so also is

$$
g=\left(1_{M} \times \alpha\right) \beta: U^{\prime} \rightarrow M \times L \times B
$$

where $U^{\prime}$ is the open subset of $U$ such that $\left(1_{M} \times \alpha\right) \beta$ is defined there. So, $\check{\tau}_{g}: \check{h}_{G}^{*}(\operatorname{Fix}(f)) \rightarrow h_{G}^{*+[M]-[N]}(B)$ is defined. Moreover, $\check{\tau}_{g}$ depends only on $f$ and not on the decomposition $f=\beta\left(1_{N} \times \alpha\right)$. Hence we define $\check{\tau}_{f}$ by

$$
\check{h}_{G}^{*}(\operatorname{Fix}(f)) \cong \check{h}_{G}^{*}(\operatorname{Fix}(g)) \stackrel{\check{\tau}_{g}}{\longrightarrow} h_{G}^{*+\rho}(B) \text {. }
$$

Again, this transfer has the properties 1.10 through 1.15 , as well as the following additional properties:

1.17. Additivity. If $V=V_{1} \cup V_{2}$ is a union of open sets such that $f_{12}=f \mid V_{1} \cap V_{2}$ (and $f$ ) is compactly fixed, then $f_{1}=f \mid V_{1}$ as well as $f_{2}=f \mid V_{2}$ are compactly fixed and

$$
\check{\tau}_{f}=\check{\tau}_{f_{1}} i_{1}^{*}+\check{\tau}_{f_{2}} i_{2}^{*}-\check{\tau}_{f_{12}} i_{12}^{*}: \check{h}_{G}^{*}(\operatorname{Fix}(f)) \rightarrow h_{G}^{*+\rho}(B),
$$

where $i_{j}: \operatorname{Fix}\left(f_{j}\right) \rightarrow \operatorname{Fix}(f)$ and $i_{12}: \operatorname{Fix}\left(f_{12}\right) \rightarrow \operatorname{Fix}(f)$ are the inclusions. 
1.18. Multiplicativity. If $f: V \rightarrow M \times E$ and $f^{\prime}: V^{\prime} \rightarrow M^{\prime} \times E^{\prime}, V \subset N \times$ $E, V^{\prime} \subset N^{\prime} \times E^{\prime}$, are both compactly fixed over $B$, then $f \times{ }_{B} f^{\prime}: V \times{ }_{B} V^{\prime} \rightarrow$ $(M \times E) \times{ }_{B}\left(M^{\prime} \times E^{\prime}\right)=\left(M \oplus M^{\prime}\right) \times\left(E \times{ }_{B} E^{\prime}\right)$ is also compactly fixed. Also $\operatorname{Fix}\left(f \times_{B} f^{\prime}\right) \approx \operatorname{Fix}(f) \times{ }_{B} \operatorname{Fix}\left(f^{\prime}\right)$ and

$$
\check{\tau}_{f \times x_{B} f^{\prime}}\left(z \times z^{\prime}\right)=\check{\tau}_{f}(z) \smile \check{\tau}_{f^{\prime}}\left(z^{\prime}\right),
$$

where $z \in \check{h}_{G}^{*}(\operatorname{Fix}(f)), z^{\prime} \in \check{h}_{G}^{*}\left(\operatorname{Fix}\left(f^{\prime}\right)\right)$.

1.19. $h_{G}^{*}(B)$ as a ground ring. $\tau_{f}^{X}: h_{G}^{*}(X) \rightarrow h_{G}^{*+\rho}(B)$, as well as $\check{\tau}_{f}: \check{h}_{G}^{*}(\operatorname{Fix}(f))$ $\rightarrow h_{G}^{*+\rho}(B)$ are both homomorphisms of $h_{G}^{*}(B)$-modules, i.e.

$$
\tau_{f}^{X}\left(x \smile p^{*}(b)\right)=\tau_{f}^{X}(x) \smile b
$$

if $p: X \rightarrow B$ is the restriction of $N \times E \rightarrow B$, and analogously for $\check{\tau}_{f}$.

This follows from the fact that all maps inducing $\tau_{f}^{X}$ are maps over $B$.

As a particular case of 1.19 (by taking $x=1 \in h_{G}^{0}(X)$ ), we obtain that the composition

$$
h_{G}^{*}(B) \stackrel{p^{*}}{\longrightarrow} h_{G}^{*}(X) \stackrel{\tau_{f}^{x}}{\longrightarrow} h^{*+\rho}(B)
$$

is multiplication by $\tau_{f}^{X}(1) \in h_{G}^{\rho}(B)$, as is also the composition

$$
h_{G}^{*}(B) \stackrel{p^{*}}{\longrightarrow} \check{h}_{G}^{*}(\operatorname{Fix}(f)) \stackrel{\check{i}_{f}}{\longrightarrow} h_{G}^{*+\rho}(B)
$$

multiplication by $\check{\tau}_{f}(1) \in h_{G}^{\rho}(B)$.

\section{THE EQUIVARIANT INDEX}

In this section, we define the index and show some of its properties. For a situation such as (1.2) we have

2.1. Definition. For any $G$-invariant neighborhood $X$ of $\operatorname{Fix}(f)$, the homomorphism

$$
I_{f}: h_{G}^{*}(B) \rightarrow h_{G}^{*}(X) \stackrel{\tau_{f}^{X}}{\longrightarrow} h_{G}^{*+\rho}(B),
$$

where the first arrow is induced by the projection $X \rightarrow B$, does not depend on $X$ and is called the index homomorphism of $f$. If we assume that $h_{G}^{*}$ is a multiplicative theory, then $h_{G}^{0}(B)$ is a commutative ring with 1 and $I_{f}$ is a homomorphism of modules over $h_{G}^{0}(B)$. Therefore, $I_{f}$ is completely determined by the element

$$
I(f)=I_{f}(1) \in h_{G}^{[M]-[N]}(B)
$$

which we call the index of $f .2 .2$ and 2.3 are generalizations of the corresponding concepts in $\left[\mathrm{Do}_{2}\right.$ and $\left.\mathrm{Pr}_{1}\right]$ and as in those cases, we have corresponding properties of $I_{f}$ and $I(f)$, which follow from those of the transfer. We list some of them. 
2.4. Localization in $E$. If $W$ is open in $V$ and $\operatorname{Fix}(f) \subset W$, then $f \mid W$ is compactly fixed and $I_{f \mid W}=I_{f}$, as well as $I(f \mid W)=I(f)$.

2.5. Localization in $B$. The index homomorphism factors as follows:

$$
h_{G}^{*}(B) \rightarrow h_{G}^{*+[M]-[N]}(B, A) \rightarrow h_{G}^{*+[M]-[N]}(B)
$$

for any closed invariant subspace $A$ of $B$ such that $\operatorname{Fix}(f) \subset N \times L \times(B-A)$. Hence the index $I(f)$ lives, in fact, in $h_{G}^{*+[M]-[N]}(B, A)$.

2.6. We have also the properties Additivity, Units, Pullbacks, Homotopy Invariance and Commutativity, which follow directly from 1.17., 1.10., 1.11., 1.13. and 1.15 ., respectively, as one easily checks. The reader should figure out the corresponding statements.

2.7. Remark. An equivariant cohomology theory which qualifies for defining the index is equivariant stable cohomotopy (see e.g. [Ko]) or its fixed point theoretical version $G$-FIX ${ }^{*}$. There, the index is a complete invariant up to homotopy and every element in the theory is realizable as the index of a suitable situation. In fact, for $G$-FIX ${ }^{*}$ we have $I(f)=[f] \in G$-FIX ${ }^{[M]-[N]}(B)$ (see [Ul or $\left.\left.\operatorname{Pr}_{2}\right]\right)$.

2.8. Remark. Let $B=\{*\}$ and $E=\mathbf{R}^{n}$ (with the trivial action of $G$ ). Given an equivariant map

$$
N \times \mathbf{R}^{n} \supset V \stackrel{g}{\longrightarrow} M \times \mathbf{R}^{n}
$$

such that $g^{-1}(0)$ is compact (i.e. if $g$ can be extended to the closure $\bar{V}$, then it has no zeroes on the boundary of $V$ ), define the degree of $g$ by

$$
\operatorname{deg}(g)=I(i-g) \in G-\mathrm{FIX}^{[M]-[N]}(*) \cong \operatorname{Stab}_{G}\left(\mathbf{S}^{N}, \mathbf{S}^{M}\right),
$$

where $\operatorname{Stab}_{G}\left(\mathbf{S}^{N}, \mathbf{S}^{M}\right)$ denotes the group of equivariant stable homotopy classes of $G$-maps from the one-point compactification of the $G$-module $N$ into the one-point compactification of the $G$-module $M$. (It is a fact easy to prove that this degree coincides with the stabilization of the one defined in [IMV].)

2.9. Before concluding this section, let us write some formulas which allow us to compute the index in some cases. Compare [Ul] for details (which are essentially the same as for the case of degree zero). In what follows we shall assume that $B$ has trivial $G$-action. We recall the following

2.10. Theorem (cf. [U1, II.6.8]). Let $p: E \rightarrow B$ be $a$ G-ENR $_{B}$ over G-trivial base space $B$. Then there exists a finite filtration $\phi=E_{0} \subset E_{1} \subset \cdots \subset E_{r-1} \subset$ $E_{r}=E$ by closed $G$ - $\mathrm{ENR}_{B}$-subspaces (or, equivalently, by $G$-cofibrations over $B)$ such that $E_{i}-E_{i-1}$ is the union of all orbits of $E$ of a fixed type.

The following lemma will also be of interest in the sequel. 
2.11. Lemma (cf. [U1, III.5.2]). Let $f: V \rightarrow M \times E$ be a compactly fixed $G$-map over a G-trivial space $B, V \subset N \times E$, and let $D \subset E$ be a closed $G$ - $\mathrm{ENR}_{B}$-subspace such that $f(V \cap(N \times D)) \subset M \times D$. Then, the G-map $f^{D}: V^{D} \rightarrow M \times D, V^{D}=V \cap(N \times D), f^{D}=f \mid V^{D}$, is compactly fixed and the difference $I(f)-I\left(f^{D}\right)$ is the index of a compactly fixed map $f_{D}$, partially defined on $N \times(E-D)$ over $B$.

Proof. Because of the localization in $E$, 2.4, we may restrict $f$ to an open $G$-invariant $V$ such that its closure $\bar{V}$ in $N \times E$ lies properly over $B . V$ and $V^{D}$ are $G$ - $\mathrm{ENR}_{B}$-spaces, thus the inclusion $V^{D} \subset V$ is a $G$-cofibration. Hence there is a $G$-deformation $d_{t}$ of $V$ over $B$, relative to $V^{D}$, such that $d_{1}^{-1}\left(V^{D}\right)$ is a $G$-neighborhood of $V^{D}$. We may also assume that $d_{t}$ remains stationary outside of a neighborhood of $V^{D}$, by modulating it via an equivariant separating function for the closure $\bar{U}$ of a neighborhood $U$ and the complement $V-W$ of a larger neighborhood $W$ of $V^{D}$ in $V . f \circ d_{t}: V \rightarrow M \times E$ is hence a $G$-homotopy of $f$ over $B$, relative to $V^{D} \cup(V-W)$. Its fixed point set is a closed subset of $\bar{W} \times I \cup \operatorname{Fix}(f) \times I$ in $V \times I$, and since $\bar{W}$ lies properly over $B$, then it is compactly fixed. Consequently, $I\left(f d_{1}\right)=I(f)$. On the other hand, using the additivity of the index,

$$
I\left(f d_{1}\right)=I\left(f d_{1} \mid U\right)+I\left(f d_{1} \mid\left(V-V^{D}\right)\right),
$$

since the fixed points of $f d_{1}$ are either in $V^{D}$ or outside of $U$.

Finally, since the restriction of $f d_{1}$ to $U$ lands into the $G-\operatorname{ENR}_{B} D$, an easy application of the commutativity shows that its index coincides with the index of the restriction $f d_{1} \mid U^{D}: U^{D} \rightarrow M \times D, U^{D}=U \cap(N \times D)$, but this last is equal to $f^{D}$.

2.12. If $X$ is any $G$-space, $H \subset G$ is a closed subgroup and $(H)$ is its conjugation class, we shall use the following notation:

$$
\begin{array}{lll}
X^{H}=\left\{x \in X \mid G_{x} \supset H\right\}, & X^{(H)}=\left\{x \in X \mid\left(G_{x}\right) \supset(H)\right\}, \\
X^{\underline{H}}=\left\{x \in X \mid G_{x} \supsetneq H\right\}, & X^{(\underline{H})}=\left\{x \in X \mid\left(G_{x}\right) \supsetneq(H)\right\}, \\
X_{H}=\left\{x \in X \mid G_{x}=H\right\}, & X_{(H)}=\left\{x \in X \mid\left(G_{x}\right)=(H)\right\},
\end{array}
$$

where $(H) \subset(K)$ means $H$ is contained in some conjugate of $K$. Hence $X_{H}=X^{H}-X^{\underline{H}}$ and $X_{(H)}=X^{(H)}-X^{(\underline{H})}$.

The difference sets of 2.10 consist of orbits of the same type, say $H_{i} \subset G$. So, $E_{i}-E_{i-1}=E_{\left(H_{i}\right)}$, where $\left(H_{1}\right) \prec\left(H_{2}\right) \prec \cdots \prec\left(H_{r}\right)$ are the different

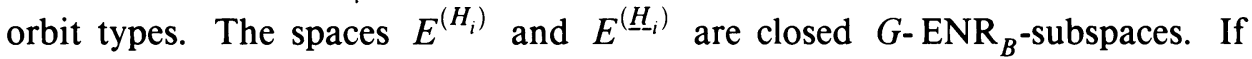
$f: V \rightarrow M \times E, V$ open in $N \times E$, is a compactly fixed $G$-map over a $G$-trivial base space $B$, and $M, N$ are trivial $G$-modules (say $M=\mathbf{R}^{m}, N=\mathbf{R}^{n}$ ), then it induces the following $G$-equivariant situations: 


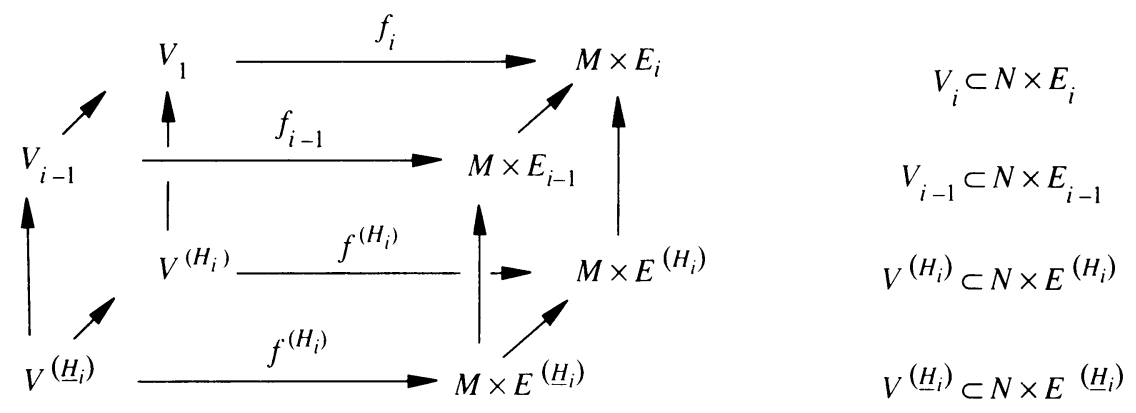

By Lemma 2.11, $I\left(f_{i}\right)-I\left(f_{i-1}\right)$ is the index of a map which we shall call $f_{\left(H_{i}\right)}$, defined on $E_{\left(H_{i}\right)}=E_{i}-E_{i-1}=E^{\left(H_{i}\right)}-E^{\left(\underline{H}_{i}\right)}$. The deformation $d_{t}$ of $V_{i}$ relative to $V_{i-1}$ gives, when restricted to $V^{\left(H_{i}\right)}$, a $G$-deformation of $V^{\left(H_{i}\right)}$ relative to $V^{\left(\underline{H}_{i}\right)}$. So, the difference $I\left(f^{\left(H_{i}\right)}\right)-I\left(f^{\left(\underline{H}_{i}\right)}\right)$ is the index of the restriction of $\left(f d_{1}\right)^{\left(H_{i}\right)}$ to $V_{\left(H_{i}\right)}$ and the shrinking of this map to $M \times E_{\left(H_{i}\right)}$ is precisely our $f_{\left(H_{i}\right)}$. Therefore

$$
I\left(f_{i}\right)-I\left(f_{i-1}\right)=I\left(f^{\left(H_{i}\right)}\right)-I\left(f^{\left(\underline{H}_{i}\right)}\right)
$$

and since $I(f)=\sum_{i} I\left(f_{i}\right)-I\left(f_{i-1}\right)$ we have

2.13. Theorem (cf. [Ul, III.5.4]). Let $f: V \rightarrow \mathbf{R}^{m} \times E$, be a compactly fixed $G$-map over a $G$-trivial base space $B$. Then

$$
I(f)=\sum\left(I\left(f^{(H)}\right)-I\left(f^{(\underline{H})}\right)\right) \in h_{G}^{m-n}(B),
$$

the sum taken over the finitely many orbit types around $\operatorname{Fix}(f)$.

\section{A LEFSCHETZ-HOPF THEOREM}

3.0. In this section we shall prove a theorem which permits us to compute the transfer in some cases as a trace of a suitable homomorphism. This theorem is a generalization of the corresponding theorem (6.18) in $\left[\mathrm{Do}_{3}\right]$ and as well, $\S 6$ and especially Lemmas (6.7), (6.10), (6.12) and (6.13) shall be used. We shall use also all notation of the preceding sections.

3.1. Theorem (cf. $\left.\left[\mathrm{Do}_{3},(6.18)\right]\right)$. Let $f: N \times V^{\prime} \rightarrow M \times E$ and let $V^{\prime} \subset E$ open and invariant, be a compactly fixed G-map over $B$ such that its image $f\left(N \times V^{\prime}\right)$ is contained in $M \times K$ for some invariant subset $K \subset V^{\prime}$ which is proper and $h_{G}$-flat over $B$. If $f$ is such that $f^{-1}(0 \times K) \cap(N \times K) \subset \mathbf{B} \times K$ for a ball $\mathbf{B} \subset N$, then the composite homomorphism

$$
\check{h}_{G}(K) \cong \check{h}_{G}(\mathbf{B} \times K) \stackrel{\check{j}}{\longrightarrow} \check{h}_{G}(\operatorname{Fix}(f)) \stackrel{\check{i}_{f}}{\longrightarrow} h_{G}(B),
$$

where $j$ : $\operatorname{Fix}(f) \subset \mathbf{B} \times K$, is given by the following trace formula

$$
\check{\tau}_{f} \check{\jmath}(a)=\operatorname{trace}\left[x \mapsto a \smile(f \mid K)^{\vee}(x)\right] \in h_{G}^{|a|+[M]-[N]}(B),
$$

where we denote by $(f \mid K)^{\vee}$ the following homomorphism of degree $[M]-[N] \in$ $R O(G)$ defined by $f: N \times K \rightarrow M \times K$

$$
\check{h}_{G}(K) \cong \check{h}_{G}((M, M-0) \times K) \rightarrow \check{h}_{G}((N, N-\mathbf{B}) \times K) \cong \check{h}_{G}(K)
$$


(since $f^{-1}(0 \times K) \cap(N \times K) \subset \mathbf{B} \times K, f$ maps $(N-\mathbf{B}) \times K$ into $\left.(M-0) \times K\right)$. In other words, the image of $a \in \check{h}_{G}(K)$ under $\check{\tau}_{f} \check{j}$ is the trace of the composite $h_{G}(B)$-endomorphism

$$
\check{h}_{G}(K) \stackrel{(f \mid K)^{\vee}}{\longrightarrow} \check{h}_{G}(K) \stackrel{a \smile}{\longrightarrow} \check{h}_{G}(K) .
$$

In particular, the index $I(f)$ equals the trace of $(f \mid K)^{\vee}: \check{h}_{G}(K) \rightarrow \check{h}_{G}(K)$.

Proof. We assume $E=L \times B$. Since $\mathbf{B} \times K \subset N \times E$ is proper there exists a tube $\mathbf{B}_{\sigma} \subset N \times E$ around $\mathbf{B} \times K$. Let $X$ be a small open neighborhood of $K$ in $V^{\prime}$ and consider the diagram

$$
\begin{aligned}
& \left(N \times E, N \times E-\mathbf{B}_{\sigma}\right) \hookrightarrow \\
& (N \times E, N \times E-\mathbf{B} \times K) \\
& \| \\
& (N, N-\mathbf{B}) \times(E, E-K) \\
& (N, N-\mathbf{B}) \times(X, X-K) \stackrel{(i-f, \text { id })}{\longrightarrow}(M, M-0) \times(E, E-0) \times{ }_{B} X \\
& \downarrow\left(\operatorname{proj}_{X}, f\right) \quad \uparrow(-\mathrm{id}) \times(d, i q) \\
& (X, X-K) \times_{B}(M, M-0) \times K \approx(M, M-0) \times(X, X-K) \times{ }_{B} K
\end{aligned}
$$

where $X$ is so small that $f$ maps $(N-\mathbf{B}) \times X$ into $(M-0) \times K$ (cf. the assumptions), and $d(x, k)=x-k, q(x, k)=k, i: K \subset X$.

The square is as in $\left[\mathrm{Do}_{3},(6.18)\right]$ not commutative, but by op. cit. (6.12) it becomes commutative after application of $\breve{h}_{G}$. Let $\xi \in h_{G}(X)$ and chase the element $s_{M} \times s_{L} \times \xi \in h_{G}^{[M]+[L]+\rho}\left((M, M-0) \times(E, E-0) \times{ }_{B} X\right)$ from the right to the left, where $s_{M} \in h_{G}^{[M]}(M, M-0), s_{L} \in h^{[L]}(L, L-0)$ are the generators. Along the upper part we get $\tau_{f}^{N \times X}\left(p^{*} \xi\right)$ where $p: N \times X \rightarrow X$ is the projection; following the bottom of the square we get

$$
\begin{gathered}
(-\mathrm{id} \times(d, i q))^{*}\left(s_{M} \times s_{L} \times \xi\right)=(-\mathrm{id})^{*}\left(s_{M}\right) \times \sum_{\nu}\left(\alpha_{\nu} \otimes\left(\beta_{\nu} \smile \check{\imath} \xi\right)\right) \\
\in h_{G}^{[M]+[L]}((M, M-0) \times(X, X-K)) \otimes_{h_{G}(B)} \check{h}_{G}^{i}(K),
\end{gathered}
$$

where $\alpha_{\nu}$ and $\beta_{\nu}$ are as in the proof of op. cit. (6.10). Hence, by op. cit. (6.7), the image of this element in $h_{G}^{[M]+[L]+i}\left(N \times E, N \times E-\mathbf{B}_{\sigma}\right) \cong h_{G}^{i+[M]-[N]}(B)$ coincides with the trace of the following endomorphism of $\breve{h}_{G}(K)$ :

$$
\begin{aligned}
x & \mapsto \sum_{\nu}(-1)^{\varepsilon_{\nu}}\left(\beta_{\nu} \smile \check{l}(\xi)\right) \gamma\left(\alpha_{\nu} \smile f\left(s_{M} \times x\right)\right) \\
& =\check{\imath}(\xi) \smile\left[\sum_{\nu}(-1)^{\varepsilon_{\nu}+\left|\beta_{\nu}\right||\xi|} \beta_{\nu} \gamma\left(\alpha_{\nu} \smile f\left(s_{M} \times \lambda\right)\right)\right]
\end{aligned}
$$

where $\gamma: h_{G}^{*}(N \times E, N \times E-\mathbf{B} \times K) \cong h_{G}^{*-[N]}(E, E-K) \rightarrow h_{G}^{*-[N]-[L]}(B)$ is (up to the isomorphism) so defined as in the proof of op. cit. (6.10). As in op. 
cit. (6.17), the bracket $[\cdots]=(f \mid K)^{\vee}(x)$ for $(f \mid K)^{\vee}$ as defined in (3.2). Thus

$$
\tau_{f}^{N \times X}\left(p^{*} \xi\right)=\operatorname{trace}\left(x \mapsto\left(\check{l}(\xi) \cup(f \mid K)^{\vee}(x)\right)\right)
$$

since $\tau_{f}^{N \times X}\left(p^{*} \xi\right)=\check{\tau}_{f} \check{\jmath}(\check{l}(\xi))$ and every $a \in \check{H}_{G}(K)$ is of the form $a=\check{l}(\xi)$ (for some small $X$ and $\left.\xi \in h_{G}(X)\right)$, the theorem follows.

3.3. Remark. The last part of the statement of the theorem, concerning the

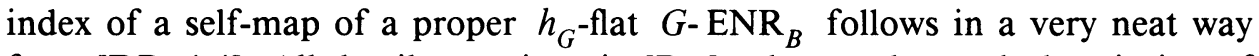
from [DP, 4.4]. All details are given in [ $\left.\mathrm{Pr}_{3}\right]$, where a thorough description of the monoidal stable homotopy category of $G-\mathrm{ENR}_{B} \mathrm{~s}$ following [DP] is also given.

3.4. Remark. $\left[\mathrm{Do}_{3},(6.17)\right]$ looks as follows

$$
s_{N} \times x=\sum_{\nu}(-1)^{\varepsilon_{\nu}+\left|\beta_{\nu}\right||\xi|} \beta_{\nu} \gamma\left[\alpha_{\nu} \smile\left(s_{M} \times x\right)\right], \quad x \in h_{G}(K) .
$$

3.5. Remark. If $N=0$ and $M \neq 0$, then one easily proves that $f$, as in 3.1, can be deformed to a fixed point free map, hence $I(f)=0$ (cf. [ $\left.\operatorname{Pr}_{1}, 4.24(\mathrm{c})\right]$ ). On the other hand, the homomorphism $(f \mid K)^{\vee}$ as in (3.2) is also zero, since the deformed map restricted to $K$ factors then through $(M-0) \times K \subset M \times K$.

3.6. Example. Let $X$ be a compact ENR and $F: I \times X \rightarrow X$ a homotopy such that the maps $f_{0}, f_{1}: X \rightarrow X ; f_{0}(x)=F(0, x), f_{1}(x)=F(1, x)$ are fixed point free. The question whether $F$ can be deformed without altering $f_{0}, f_{1}$ to a fixed point free homotopy can be answered as follows. $F$ obviously determines a compactly fixed map $F: \mathbf{R} \times X \rightarrow X$. The obstruction to deform $F$ as wanted is $I(F) \in h^{-1}(*)$ for each cohomology theory $h^{*}$. By the Lefschetz-Hopf Theorem 3.1,

$$
I(F)=\operatorname{trace}\left(\sigma F^{*}: h^{*}(X) \stackrel{F^{*}}{\longrightarrow} h^{*}((I, \partial I) \times X) \cong h^{*-1}(X)\right) .
$$

In particular, if we take $h^{*}=\pi_{s}^{*}$, stable cohomotopy, the obstruction can be computed as a trace lying in $\mathbf{Z}_{2}$. (This answers a question posed by Geoghegan.)

\section{Comments}

4.0. We shall shortly sketch what the corresponding ideas should be if we use Becker and Gottlieb's treatment, instead of Dold's approach to the transfer.

We shall be interested in $G$-fibrations $p: E \rightarrow B$ such that $B$ is a finite dimensional $G$-complex and each fiber is $H$-homotopy equivalent to a finite $H$-complex, for $G$ a compact Lie-group, $H$ a suitable subgroup (see [Wa]) of $G$. The situations we are interested in are commutative triangles

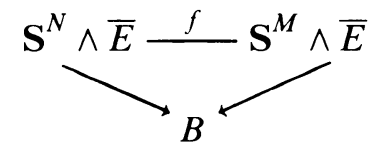

where $\mathbf{S}^{M}, \mathbf{S}^{N}$ are the one-point compactifications of the real representations $M, N$ and $\bar{E}=E \cup B$. Here $\mathbf{S}^{M} \wedge \bar{E}$ is short for $\left(\mathbf{S}^{M} \times B\right) \wedge_{B} \bar{E}$, where $\wedge_{B}$ is 
smash product of ex-spaces of $B$ and $\mathbf{S}^{M} \times B$ is seen as an equivariant ex-space with the natural section at $\infty$. For simplicity we only write $\wedge$ instead of $\wedge_{B}$.

In order not to make the section too long, we shall consider the nonequivariant case, although with the due care it carries over to the general case as already done by Waner [Wa] or by Lewis et al. [LMM] in the case $M=N=0$. We shall here use freely all duality results of [BG].

Hence, for $M=\mathbf{R}^{m}, N=\mathbf{R}^{n}$ we denote $\mathbf{S}^{M}$ and $\mathbf{S}^{N}$ simply by $\mathbf{S}^{m}$ and $\mathbf{S}^{n}$. Let us thus consider commutative triangles

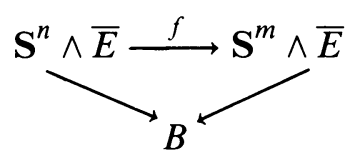

such that $f$ preserves the section.

Let $\mu: \mathbf{S}^{s} \times B \rightarrow \bar{E} \wedge \hat{E}$ be a duality map (coevaluation) and $\hat{\mu}: \bar{E} \wedge \hat{E} \rightarrow \mathbf{S}^{S} \times B$ its dual (evaluation). Consider the sequence of maps of ex-spaces

$$
\begin{gathered}
\left(\mathbf{S}^{n} \wedge \mathbf{S}^{s}\right) \times B \\
\stackrel{1 \wedge \mu}{\longrightarrow} \mathbf{S}^{n} \wedge \bar{E} \wedge \hat{E} \stackrel{(1, f) \wedge 1}{\longrightarrow} \bar{E} \wedge \mathbf{S}^{m} \wedge \bar{E} \wedge \hat{E} \\
\stackrel{1 \wedge \hat{\mu}}{\longrightarrow} \bar{E} \wedge \mathbf{S}^{m} \wedge \mathbf{S}^{s} \cong\left(\mathbf{S}^{m} \wedge \mathbf{S}^{s}\right) \wedge \bar{E}
\end{gathered}
$$

where the second arrow sends $t \wedge e \wedge \hat{e} \mapsto e \wedge f(t \wedge e) \wedge \hat{e}$.

If $A \subset B$ is a subcomplex, then the composite (4.3) maps $\mathbf{S}^{n} \wedge \mathbf{S}^{S} \times A \cup\{\infty\} \times B$ into $\mathbf{S}^{m} \wedge \mathbf{S}^{s} \times E_{A} \cup\{\infty\} \times B, E_{A}=p^{-1} A$, since all maps are ex-maps of $B$. Thus 4.3 induces a map

$$
\tau_{f}: \mathbf{S}^{n+s} \wedge(B / A) \rightarrow \mathbf{S}^{m+s} \wedge\left(E / E_{A}\right)
$$

which in cohomology defines a homomorphism

$$
\tau_{f}: h^{*}\left(E, E_{A}\right) \rightarrow h^{*+m-n}(B, A) .
$$

In particular, if $A=\phi$,

$$
\tau_{f}: h^{*}(E) \rightarrow h^{*+m-n}(B) .
$$

4.7. Remark. In light of the paper of Dold and Puppe on duality, trace and transfer [DP], one sees that (4.6) is the same homomorphism as $\tau_{f^{\prime}}^{E}$ of $\S 2$, if $p: E \rightarrow B$ is an $\mathrm{ENR}_{B}$ and

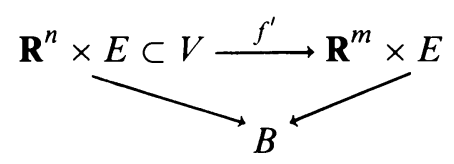

is the restriction of $f$ to $V=f^{-1}\left(\mathbf{R}^{m} \times E\right), \mathbf{R}^{m} \times E \subset \mathbf{S}^{m} \wedge \bar{E}, \mathbf{R}^{n} \times E \subset \mathbf{S}^{n} \wedge \bar{E}$. 4.8. Remark. As done in $\left[\mathrm{Pr}_{3}\right]$ one can give an alternative construction of the equivariant stable category by defining suspensions as products with $(M, M-0)$. In this category, the maps (1) and (2) in (1.3) are invertible; therefore, the transfer is given by a stable map

$$
\tau_{f}: B \rightarrow X
$$


of degree $\rho=[M]-[N] \in R O(G)$, which, as it is the case in 4.0, is represented by a map of pairs

$$
(N \times S, N \times S-0) \times B \rightarrow(M \times S, M \times S-0) \times X
$$

for a large enough $G$-module $S$. Analogously, the index homomorphism is induced by the stable map

$$
i_{f}: B \rightarrow B
$$

of degree $\rho$, which is the result of composing $\tau_{f}$ with the projection $X \rightarrow B$.

4.9. Remark. The equivariant transfer defined in $\S 1$ generates the equivariant stable homotopy category of $G$-ENRs. One may prove a generalization of Schäfer's results [Sch, Kap 5], showing that every stable map of degree $[M]-[N] \in R O(G)$, as in $\left[\operatorname{Pr}_{3}\right]$, factors through a transfer and a map in the nonstable equivariant homotopy category. Details about these results will appear elsewhere.

4.10. Example. Let $p: E=\mathbf{S}^{2} \rightarrow \mathbf{S}^{2}$ be the identity, where $\mathbf{S}^{2}=\mathbf{C} \cup\{\infty\}$ is the Riemann sphere, and let $f: \mathbf{C} \rightarrow \mathbf{C} \times \mathbf{S}^{2}$ be such that $f(z)=\left(z^{n}, z\right)$. Then, if $h^{*}$ is ordinary cohomology with Z-coefficients, the only relevant part of the transfer is

$$
\tau_{f}: H^{0}\left(\mathbf{S}^{2}\right) \rightarrow H^{2}\left(\mathbf{S}^{2}\right)
$$

and $\tau_{f}(1)=I(f)=n \in \mathbf{Z}=H^{2}\left(\mathbf{S}^{2}\right)$ (cf. $\left.\left[\operatorname{Pr}_{1}, 4.27\right]\right)$.

4.11. For the case of an infinite dimensional base space $B$ the correct set up is duality in the category of spectra, as done by Clapp in $[\mathrm{Cl}]$ or its equivariant analogue (see e.g. [DP or LMS]).

\section{REFERENCES}

[BG] J. Becker and D. Gottlieb, Transfer maps for fibrations and duality, Compositio Math. 33 (1976), 107-133.

[Cl] M. Clapp, Duality and transfer for parametrized spectra, Arch. Math. 37 (1981), 462-472.

$\left[\mathrm{Do}_{1}\right]$ A. Dold, Lectures on algebraic topology, 2nd. ed., Die Grundlehren der mathematisches Wissenschaften Einzeldarstellungen, Bd. 200, Springer-Verlag, Berlin, Heidelberg, and New York, 1972.

$\left[\mathrm{Do}_{2}\right]$ - The fixed point index of fibre-preserving maps, Invent. Math. 25 (1974), 281-297.

$\left[\mathrm{Do}_{3}\right]$ - The fixed point transfer of fibre-preserving maps, Math. Z. 148 (1976), 215-244.

[DP] A. Dold and D. Puppe, Duality, trace and transfer, Proc. Internat. Conf. on Geometric Topology, PWN, Warszawa, 1980, pp. 81-102.

[IMV] J. Ize, I. Massabò, and A. Vignoli, Degree theory for equivariant maps. I, Trans. Amer. Math. Soc. 315 (1989), 433-510.

[Ko] C. Kosniowski, Equivariant cohomology and stable cohomotopy, Math. Ann. 210 (1974), 83-104.

[LMM] G. Lewis, P. May, and J. McClure, Ordinary $R O(G)$-graded cohomology, Bull. Amer. Math. Soc. (N.S.) 4 (1981), 208-212.

[LMS] G. Lewis, P. May, and M. Steinberger, Equivariant stable homotopy theory, Lecture Notes in Math., vol. 1213, Springer-Verlag, Berlin, Heidelberg, and New York, 1986. 
[Pr $\left.{ }_{1}\right]$ C. Prieto, Coincidence index for fiber-preserving maps: An approach to stable cohomotopy, Manuscripta Math. 47 (1984), 233-249.

$\left[\mathrm{Pr}_{2}\right] \quad K O(B)$-graded stable cohomotopy over $B$ and $R O(G)$-graded G-equivariant cohomotopy: A fixed point theoretical approach to the Segal conjecture, Contemp. Math. 58 (1987), 89-107.

$\left[\mathrm{Pr}_{3}\right] \quad$, Una fórmula de Lefschetz-Hopf para el indice de coincidencia equivariante parametrizado, Aportaciones Mat.: Comun. 5 (1988), 73-88.

[Sch] B. Schäfer, Fixpunkttransfer für stetige Familien von ANR-Räumen; Existenz und axiomatische Characterisierung, Doktorarbeit, Heidelberg, 1981.

[U1] H. Ulrich, Fixed point theory of parametrized equivariant maps, Lecture Notes in Math., vol. 1343, Springer-Verlag, Berlin, Heidelberg, and New York, 1988.

[Wa] S. Waner, Equivariant fibrations and transfer, Trans. Amer. Math. Soc. 258 (1980), 369-384.

Instituto de Matemáticas, U.N.A.M., Ciudad Universitaria, 04510 México, D.F. MeXico E-mail address: IMATE@UNAMVM1.bitnet

IBM Deutschland GmbH, Entwicklung und Forschung, 7030 Böblingen, Germany 